\title{
Transcranial magnetic stimulation for the treatment of cocaine addiction: evidence to date
}

This article was published in the following Dove Press journal: Substance Abuse and Rehabilitation

\section{Corinna Bolloni \\ Paola Badas \\ Giorgio Corona \\ Marco Diana \\ Laboratory of Cognitive \\ Neuroscience, G Minardi Department \\ of Chemistry and Pharmacy, \\ University of Sassari, Sassari, Italy}

Correspondence: Corinna Bolloni; Marco Diana

Laboratory of Cognitive Neuroscience,

G Minardi Department of Chemistry and Pharmacy, University of Sassari, 23A Via

Muroni, Sassari 07100, Italy

Tel +39079 228711

Email corinnabolloni@gmail.com; dsfdiana@uniss.it

\begin{abstract}
There is a common consensus in considering substance-use disorders (SUDs) a devastating chronic illness with social and psychological impact. Despite significant progress in understanding the neurobiology of SUDs, therapeutic advances have proceeded at a slower pace, in particular for cocaine-use disorder (CUD). Transcranial magnetic stimulation (TMS) is gaining support as a safe and cost-effective tool in the treatment of SUDs. In this review, we consider human studies that have investigated the efficacy of TMS in achieving therapeutic benefits in treating CUD. All studies conducted to date that have evaluated the therapeutic effect of TMS in CUD are included. We focus on the protocol of stimulation applied, emphasizing the neurophysiological effects of coils employed related to outcomes. Moreover, we examine the subjective and objective measurements used to assess the therapeutic effects along the timeline considered. The revision of scientific literatures underscores the therapeutic potential of TMS in treating CUD. However, the variability in stimulation protocols applied and the lack of methodological control do not allow us to draw firm conclusions, and further studies are warranted to examine the interaction between TMS patterns of stimulation relative to clinical outcomes in depth.
\end{abstract}

Keywords: TMS, cocaine-use disorder, PfCx, craving, intake, dopamine

\section{Introduction}

The annual overview of the European drug situation highlighted that substance-use disorders (SUDs; ie, cannabis, cocaine, MDMA, amphetamines, opioids) represent a major public health concern in the Western world, with about 24.3 million young adult users (aged 15-34 years) in 2017. ${ }^{1}$ SUDs can be described as a chronic neuropathology characterized by a reduction in the ability to control compulsive drug-seeking behavior, regardless of negative consequences. ${ }^{2}$ Preclinical studies in rodents and human imaging evidence have shown the role of repetitive drug use in aberrant forms of neural plasticity consisting, briefly, in a significant reduction in dopaminergic activity linked to the dysfunction of cortical and subcortical pathways. ${ }^{3-21}$ These findings complement the fundamental building blocks of the dopamine (DA) hypothesis of drug addiction, which ascribes to the hypofunctioning DA system a key role in the genesis of drug abuse and leads to the theory that functional "boosting" of DA signaling may hold beneficial effects in reducing drug craving/intake., ${ }^{4,22,23}$

Drug addiction is now considered a whole-brain pathology, since brain-wide activity patterns are compromised. ${ }^{18,19,24,25}$ These changes in brain function lead to the 
motivational and cognitive impairment that characterizes addictive disorders. ${ }^{10,13,26}$ Indeed, alterations in mesocortical brain networks and related dysfunction in dopaminergic activity are accompanied by increased salience for the abused substance and increased cue reactivity for related stimuli, which in turn support the motivation/drive to use the substance and to relapse. ${ }^{27-30}$ This mechanism seems to have a putative role in the development of addiction, amplified and supported by an uncontrollable urge to take the substance of abuse (ie, craving), which leads to searching for the substance and relapse. Indeed, higher craving rates are related to higher relapse rates. ${ }^{18,19,31,32}$ Despite the fact that drug craving is considered an important risk factor for relapse, it can be counteracted and restricted by exerting cognitive and behavioral control. ${ }^{33,34}$ However, the diminished functioning of the dorsolateral prefrontal cortex (D1PfCx) and anterior cingulate cortex provides a reason for the impaired inhibitory behavioral control and higher tendency to relapse in alcohol and/or drug use. ${ }^{34-38}$

However, despite these recent advances in understanding the neurobiology of addiction, expectations of therapeutic treatments have fallen shorter than desired. ${ }^{39,40}$ Transcranial magnetic stimulation (TMS) represents a nonpharmacological tool and a testable opportunity in the treatment of SUDs, owing to its capacity to target and modulate specific brain circuits that are involved in the neuropathology of addiction. $^{41-44}$

Therefore, we searched among published studies that investigated the efficacy of TMS in the treatment of SUDs and focused on those applied to cocaine addicts. Papers were identified through NCBI PubMed research by using "TMS", "addiction", and "cocaine" as keywords. Among published studies, only six focused on the treatment of cocaine addiction. These are reviewed in the present paper, with particular reference to the stimulation protocol applied and outcome observed.

\section{TMS physiology}

Since 1985, when TMS was implemented for the first time in the study of motor-cortex excitability, the potential therapeutic effect of brain stimulation is being investigated in different psychiatric disorders, such as major depression, obsessive-compulsive disorders, schizophrenia, and addictive disorders. ${ }^{41,43-50}$ TMS can be described as a nonsurgical brain stimulation that is able to modulate cortical excitability through magnetic fields inducted over the scalp. ${ }^{51}$ The passage of electric current in the coil induces a transient, high-intensity magnetic pulse that penetrates the scalp and reaches the neurons of the targeted cortical area painlessly in the conscious subject. Generally, $1 \mathrm{~Hz}$ frequency (or below) of consecutive stimuli, ie, repetitive TMS (rTMS), inhibits cortical excitability, whereas high frequency (5-20 $\mathrm{Hz}$ ) produces increased cortical excitability. ${ }^{43,44}$ This change in cortical activity is able to produce both physiologic and behavioral effects, depending on the parameters of stimulation applied. ${ }^{52-54}$ The total duration of the session, the frequency of stimulation employed, the intensity (relative to motor threshold [MT]), and pattern of stimulation are the key factors in determining long-lasting TMS effects, since physiological and behavioral effects are mediated by the traditional phenomena of Hebbian synaptic plasticity, consisting in longterm potentiation (LTP) and long-term depression (LTD) of neuronal activity. ${ }^{44,55,56}$ Despite variability in the protocol of stimulation applied, a number of studies (Table S1) have shown efficacy of TMS in reducing craving and consumption in alcoholics and nicotine-dependent subjects. Additional recent work highlights the therapeutic potential of rTMS in cue-induced craving for methamphetamine, heroin-cue craving, and food craving. ${ }^{57-65}$

These findings suggest that rTMS modulates neural activity via two main mechanisms: through the frontostriatal glutamate-bearing afferents to medium spiny neurons of the ventral striatum, and projections from pyramidal neurons of the fifth layer of the PfCx impinging upon DA-containing midbrain neurons, thereby inducing DA release in the nucleus accumbens. ${ }^{11,22,66,67}$

The effect of TMS to increase DA levels transiently in cortical areas and its ability to modulate reduced dopaminergic activity in the limbic system appears to be among the mechanisms in restoring predrug functionality at a system level. ${ }^{44}$ On the other hand, by stimulating the PfCx, functioning of the cortical network can be strengthened and hopefully improve executive-control circuits. . $^{7,935,68}$

\section{Key TMS factors}

At present, the need to find the best stimulation parameters to optimize the clinical outcome is the main target. Recent studies have pointed out the importance of the characteristics of the magnetic E-field inducted in association with the clinical outcome, theorizing that a deeper stimulation may have more chance of reaching dysfunctional brain areas, and through this "direct way" better odds of obtaining long-lasting effects, such as reduction in craving and drug consumption. ${ }^{69-71}$

The activation of targeted brain areas depends on the shape of the magnetic E-field, spatial accuracy, and 
penetration depth of stimulation. ${ }^{72}$ These factors are dictated by coil geometry, which then defines the capability of stimulation to activate the brain areas of interest aimed at restoring the dysfunctional neuronal network, eventually. Considering that the intensity of the E-field is maximal closest to the coil, stimulation intensity is strongest over the brain surface and rapidly attenuates with distance..$^{73}$ The most widely used coil implemented in the study of TMS effects is the figure-of-eight coil, which owing to its geometry (figure of eight), is able to project focal fields over the brain surface with high spatial resolution $\left(2.5-3 \mathrm{~cm}^{2}\right) \cdot{ }^{74}$ However, the E-fields remain quite superficial and tend to dissipate in intensity rapidly, with little chance of reaching deeper brain areas disrupted by the neuropathology of addiction. ${ }^{73}$ Moreover, discrepancies in the geometry of magnetic fields generated by the figure-ofeight coil and thus in the localization of the area targeted may account for the variability in effects observed.

In 2002, Roth et al designed a new coil called a Hesed-coil (H-coil) able to stimulate the PfCx bilaterally and through the stimulation of frontostriatal projections activate midbrain DA neurons and other subcortical areas involved in dopaminergic activity, at least in theory. ${ }^{69,75}$ Recently, Malik et al tested the capacity of the H-coil to modulate DA release in eight healthy subjects, finding that low-frequency rTMS $(1 \mathrm{~Hz})$ significantly decreased DA levels in the substantia nigra and sensorimotor and associative striata. ${ }^{76}$ This novel coil seems to have more chance of obtaining consistent and prolonged effects, presumably due to its ability to stimulate subcortical brain regions that are involved in the development of addiction's neuropathology. However, since cortical thickness is contained within $2 \mathrm{~cm}$ from the skin surface, targeting fifth-layer cortical pyramidal neurons (efferent) may suffice to modulate subcortical circuitries involved in taking and relapse. ${ }^{22,44,77}$

If the coil geometry is among the key factors in the efficacy of stimulation to activate the targeted brain areas, anatomical pathways also play an important role in the final effect of stimulation. ${ }^{53,72}$ For this reason, the TMS navigator device integrated with a magnetic resonance imaging guide was developed, which is able to identify the targeted area to stimulate and to estimate the orientation, intensity, and gradients of the E-fields based on the subject's brain anatomy. ${ }^{78}$ This innovative integrated approach of TMS plus neuronavigation will be useful in future to optimize the TMS effect and by that to ameliorate clinical benefits.

\section{TMS for treatment of cocaine addiction: evidence to date}

The six studies that investigated the effect of TMS in cocaine addiction are discussed herein and summarized in Table 1. In 2007, Camprodon et al investigated the effect of rTMS on cocaine craving. Secondary end points were changes in anxiety, happiness, sadness, and discomfort. ${ }^{79}$ In this randomized crossover study, six patients fulfilling Diagnostic and Statistical Manual of Mental Disorders, 4th edition (DSM)-IV criteria for cocaine dependence underwent two session of rTMS - one to the left and one to the right DlP$\mathrm{fCx}$ - with a week's break between sessions. Patients were asked to complete a visual analog scale (VAS) ranging from "not at all" to "more than ever" before, immediately after, and 4 hours after TMS. Notably, during the whole study, patients remained hospitalized. The protocol of stimulation

Table I Studies that have implemented TMS in the treatment of cocaine addicts

\begin{tabular}{|c|c|c|c|c|c|c|}
\hline & $\mathbf{n}$ & TMS device and parameters & $\begin{array}{l}\text { Target } \\
\text { area }\end{array}$ & $\begin{array}{l}\text { Control } \\
\text { group }\end{array}$ & Assessment & Results \\
\hline Terraneo et $\mathrm{al}^{85}$ & 32 & $\begin{array}{l}\text { rTMS (8-coil), } 8 \text { sessions, } 40 \text { trains, } \\
\text { I5 Hz, I00\% MT, } 2,400 \text { pulses }\end{array}$ & DIPfCx, left & Pharm & Urine, craving & $\begin{array}{l}\text { More cocaine-free urine samples in } \\
\text { rTMS group, reduction in craving in } \\
\text { rTMS group }\end{array}$ \\
\hline Rapinesi et $a^{86}$ & 7 & $\begin{array}{l}\text { rTMS (H coil), } 12 \text { sessions, } 20 \text { trains, } \\
20 \mathrm{~Hz}, 100 \% \text { MT, } 720 \text { pulses }\end{array}$ & $\begin{array}{l}\text { DIPfCx, } \\
\text { bilateral }\end{array}$ & No & Craving (VAS) & Reduction in craving \\
\hline Bolloni et $\mathrm{al}^{87}$ & 10 & $\begin{array}{l}\text { rTMS (HI coil), I } 2 \text { sessions, } 20 \text { trains, } \\
10 \mathrm{~Hz}, 100 \% \mathrm{MT}, \mathrm{I}, 000 \text { pulses }\end{array}$ & $\begin{array}{l}\text { DIPfCx, } \\
\text { bilateral }\end{array}$ & Sham TMS & Hair analysis & $\begin{array}{l}\text { Reduction in Intake in } 10 \mathrm{~Hz} \\
\text { rTMS group, no difference among } \\
\text { subjects }\end{array}$ \\
\hline Politi et $\mathrm{al}^{81}$ & 36 & $\begin{array}{l}\text { rTMS (8-coil), I0 sessions, } 20 \text { trains, } \\
\text { I5 Hz, I00\% MT, } 600 \text { pulses }\end{array}$ & DIPfCx, left & No & Craving (VAS) & Reduction in craving \\
\hline $\begin{array}{l}\text { Camprodon } \\
\text { et } \mathrm{al}^{79}\end{array}$ & 6 & $\begin{array}{l}\text { rTMS, } 2 \text { sessions, } 20 \text { trains, } 10 \mathrm{~Hz}, 90 \% \\
\text { MT, } 2,000 \text { pulses }\end{array}$ & $\begin{array}{l}\text { DIPfCx, } \\
\text { right/left }\end{array}$ & No & Craving (VAS) & $\begin{array}{l}\text { Reduction in craving with right } \\
\text { rTMS }\end{array}$ \\
\hline Hanlon et $\mathrm{al}^{92}$ & $\mathrm{II}$ & $\begin{array}{l}\text { cTBS + fMRI, I session, I I0\% MT, I,800 } \\
\text { pulses }\end{array}$ & MPfCx, left & Sham TMS & Craving & Reduction in craving \\
\hline
\end{tabular}

Abbreviations: rTMS, repetitive transcranial magnetic stimulation; MT, motor threshold; cTBS, continuous $\theta$-burst stimulation; fMRI, functional magnetic resonance imaging; DIPfCx, dorsolateral prefrontal cortex; MPfCx, medial prefrontal cortex; VAS, visual analogue scale. 
consisted in two sessions of stimulation (right/left) with 20 trains each of 10-second length and 1 second of interstimulus for a total of 2,000 pulses. The frequency of stimulation was $10 \mathrm{~Hz}$, while the intensity was $90 \%$ of the individual's MT. The authors found a transient effect of one session of $10 \mathrm{~Hz}$ rTMS over the right DIPfCx, but not the left, which consisted in a (statistically) significant reduction in craving for cocaine. A significant difference in "desire to consume cocaine" was found between baseline and post-TMS ratings craving and between post-TMS and 4 hours post-TMS, but no significant difference was found between pre-TMS and 4 hours later. Despite the TMS effect resolving in 4 hours after stimulation, this research provided the first evidence that one session of high-intensity rTMS over the right DIPfCx transiently reduce craving in chronic cocaine abusers. ${ }^{79,80}$ Following this preliminary study, Politi et al explored the potential of rTMS in reducing cocaine craving by applying it over the left DIPfCx for ten daily sessions. High-frequency (15 Hz) rTMS was administered over the left DIPfCx of 36 cocaine addicts. ${ }^{81}$ The stimulation consisted of 20 trains of rTMS at $100 \%$ subjective MT for a total of 600 pulses. No control group was considered in this experiment. As in the previous study, the researchers assessed the TMS effects through the VAS, and found a reduction in craving level. Although different in the paradigm applied, these two studies suggest that rTMS reduces cocaine craving. Moreover, they demonstrated the central role of DlPfCx in modulating craving, as suggested earlier by imaging studies. ${ }^{82-84}$

Terraneo et al studied the effects of rTMS on cocaine intake by applying it over the left DIPfCx. ${ }^{85}$ The authors assessed the rTMS effect with an objective marker of cocaine consumption. The primary outcome of the study was the use of cocaine assessed by urine drug screen. Secondary outcomes were cocaine craving and depressive symptoms. Unlike previous studies, the authors described the stimulator device used and the procedure to obtain subjective MT. They employed a figure-of-eight coil (as per Camprodon et al and Politi et al) to deliver the treatment, which consisted of eight sessions of rTMS delivered at $15 \mathrm{~Hz}$ and $100 \%$ MT, 60 pulses per train, and 40 stimulation trains, with 15 seconds of interstimulus interval for a total of 2,400 pulses. They used a TMS Navigator. This approach allows the experimenter to have higher methodological control. The experiment was designed in two stages, and included a control group treated with a routine pharmacological protocol consisting in pramipexole $0.35 \mathrm{mg}$ three times daily, bupropion $150 \mathrm{mg}$ daily, oxazepam $15 \mathrm{mg}$ three times daily, triazolam $0.25 \mathrm{mg}$ daily, and $\gamma$-hydroxybutyrate $1.75 \mathrm{~g}$ daily. ${ }^{85} \mathrm{~A}$ total of 32 cocaine addicts were randomly assigned to the experimental group (rTMS over left DIPfCx) or the control group during 29 days. The rTMS treatment was applied with daily sessions for the first 5 days, and once a week for the following 3 weeks. At the end of stage 1, a 63-day follow-up took place, during which the participants could choose to continue in the same group or switch to the other. During stage 2, participants belonging to control group in stage 1 received the same protocol of stimulation applied in the rTMS experimental group. At the end of stage 1,16 patients $(100 \%)$ concluded the treatment in the rTMS group, while 13 patients $(81 \%)$ concluded the pharmacological treatment in the control group. The authors analyzed the urine drug tests in the two groups at the end of stage 1, finding a higher number of cocaine-free urine samples in the rTMS group. Similar observations were found in the craving VAS, which resulted in significantly lower scores in the experimental group. The ten subjects that switched to the rTMS group in stage 2 displayed significant improvement with favorable outcomes comparable to those of the rTMS group. No differences were found in secondary outcomes. In spite of the open-label design and the use of a variety of pharmacological treatments as a control group, this study provided significant progress in methodological control, since an objective marker of cocaine consumption was used. Another important aspect of the Terraneo et al study is the direct comparison between rTMS-treated patients and pharmacological treatment (as usual) patients. ${ }^{85}$ Rapinesi et al administered 12 sessions of rTMS through the H-coil to seven cocaine addicts. ${ }^{86}$ The 12 sessions were applied three times a week alternately during 4 weeks. Bilateral rTMS was delivered at high frequency $(15 \mathrm{~Hz}$ ) and $100 \%$ MT in 20 trains with 2 seconds of interstimulus interval for a total of 8,640 pulses ( 720 pulses/session). They used cocaine craving measured with VAS as an outcome assessed the week before, each week during the treatment and 1 month after rTMS. At the end of the study, the authors found a significant craving reduction from baseline to 2 weeks and 4 weeks and from baseline to 1 month later. Despite there being a significant increase of craving from week 4 to 8 , this study provided the first evidence of medium-term effect (baseline to 1 month after treatment) of bilateral rTMS of the PfCx as a whole in cocaine craving. However, the small sample, absence of a control group, and subjective outcome did not allow the authors to make any definitive conclusion. In 2016, we designed a double-blind randomized pilot study to test the efficacy of bilateral deep rTMS in cocaine intake. ${ }^{87} \mathrm{~A}$ total of 18 treatment-seeking patients with current CUD according to DSM-IV criteria were recruited and randomly assigned to 
active $10 \mathrm{~Hz}$ stimulation or sham stimulation. A total of 12 sessions of rTMS were administered three times/week (every other day) for 4 weeks at $100 \%$ MT over the bilateral PfCx. Each session comprised 20 trains of 50 pulses with $15 \mathrm{sec}-$ onds of interstimulus interval between trains, for a total of 1,000 pulses per session. ${ }^{88,89}$ The activation of the sham protocol by a magnetic card reader mimicked the acoustic sounds of the active one without inducing magnetic fields. We considered as outcome the cocaine intake assessed by hair analysis before treatment and at 1,3, and 6 months later. We decided to use the hair test as a measure of drug intake, because it provides long-term information on drug consumption with higher sensitivity and specificity than urine analysis. ${ }^{90,91}$ We also monitored the subjects who abandoned the study before the end of treatment (dropouts) and relapses among those patients who completed the protocol. Three subjects from the sham group (37\%) and one from the active group (10\%) abandoned the study before ending the 12 sessions of stimulation. Two subjects of four $(50 \%)$ from the sham group relapsed three times after the treatment, while two subjects of six (33\%) from the active group reported one relapse after 6 months from onset of treatment. At the end of the study we analyzed the effect of deep rTMS on cocaine intake on ten cocaine addicts over a 6-month period. Betweengroup analysis indicated no difference on cocaine intake along time; however, the exploratory within-group analysis showed a significant reduction in the amount of cocaine detected from baseline to 3 and 6 months later in the active group and not in the sham one. Notably a lower rate of dropout was observed in the active group compared to the sham. In contrast to previous studies, we tested the effect of TMS in both active and sham groups, finding a profound reduction in cocaine intake in both but no difference in cocaine intake between the two experimental condition, thereby highlighting a strong placebo effect. ${ }^{79,81,85,86}$ However, the paucity of the sample may have played a role in these results. Moreover, we used as outcome an objective measure of cocaine intake (hair analysis) over 6 months. Cocaine craving was not measured. In 2015, Hanlon et al, starting from a new theoretical model to choose the target area to be stimulated, applied continuous $\theta$-burst stimulation (cTBS) over the medial prefrontal cortex (MPfCx). ${ }^{92}$ Unlike classic rTMS, which delivers several trains of consecutive stimuli, cTBS delivers bursts of three pulses at $50 \mathrm{~Hz}$ applied at $5 \mathrm{~Hz}$ at an amplitude determined by subjective MT. cTBS is expected to induce LTD (whereas intermittent TBS should produce LTP) in a given brain area, and similar effects have been observed in humans by using continuous or intermittent TBS, respec- tively. ${ }^{93-96}$ On these bases, Hanlon et al performed a singleblind, sham-controlled, crossover study to test the efficacy of cTBS over MPfCx to modulate craving in cocaine-dependent subjects. ${ }^{92}$ Craving was evaluated through self-report (score 0-10) three times before and three times after cTBS sessions. They recruited eleven chronic cocaine users, who underwent behavioral assessment and urine drug screens before treatment with TMS-blood-oxygen-level dependent (BOLD) scanning. Then, each subject underwent both real and sham rTMS administered over FP1 (landmark based on an EEG 10-20 system). Subsequently, another TMS/BOLD scan was acquired. To obtain an estimate of LTD induced by cTBS, the researchers compared the evoked BOLD signal after real/sham stimulation to the evoked BOLD signal pre$\mathrm{real} / \mathrm{sham} \mathrm{cTBS}$, and the same procedure was applied for craving. The results showed lower TMS-evoked activity in the cortical area near the coil (MPfCx), as well as in projections to arginine vasotocin after real cTBS and a greater reduction than sham cTBS in the Insula, middle temporal gyrus, thalamus, and caudate. Moreover, they found an increase in craving after sham stimulation, but no significant difference in mean scores was found between the two groups.

Despite the small sample, this study offered a new contribution and perspective to the stimulation models implemented, since instead of stimulating the DIPfCx network and amplifying executive cortical control through LTP plasticity, they targeted the MPfCx, in order to attenuate limbic drive circuits through LTD-like plasticity. Further, Hanlon et al reported similar encouraging results on the $\mathrm{MPfCx} \cdot{ }^{97}$

\section{Discussion}

In this paper, we reviewed studies that investigated the potential of rTMS in the treatment of cocaine addiction. We reviewed protocols of stimulation delivered, outcomes used, and results observed. Despite CUD representing a word health emergency associated with high relapse rates (short-term relapse rates can reach 75\%) significant disability, and substantial mortality, only six studies had explored the effects of TMS in CUD. ${ }^{98}$ Moreover, there has been no US Food and Drug Administration-approved pharmacotherapy to date, and behavioral approaches to chronic cocaine use have had limited success.

Studies that investigated the therapeutic potential of TMS had generally small samples, were variable in the protocol of stimulation applied, and different in the measurement of outcomes considered. An important issue concerns the study design applied. From this revision of the literature, it emerged that only two studies provided a sham control group, 
an element that maximizes methodological control, allowing evaluation of the placebo effect so as to derive the real effect of treatment implemented (ie, TMS). In terms of stimulation paradigms, studies differed in both coil employed and parameters adopted. The majority of rTMS studies in CUD have used the figure-of- 8 coil to target the DIPfCx (left side), a central node in the frontostriatal network whose functionality is inhibited in addiction disorders. ${ }^{8,9}$ Cortical dysfunction may account at least in part for the impaired executive control that is required to resist drug-related stimuli and cease drug-seeking behavior. For this reason, papers reviewed here aimed at prefrontal brain regions, in order to enhance cortical activity, improve behavioral control, and through this inhibit drug intake. From a neurobiological perspective, the clinical beneficial effects of TMS over DIPfCx are supported by the evidence, which demonstrated the enhancement of dopaminergic activity in the midbrain (nucleus accumbens), which is depressed in addiction. ${ }^{64,65-99}$ Two studies implemented the $\mathrm{H}$-coil in the treatment of cocaine addiction, finding a significant reduction in craving and intake. ${ }^{86,87}$ However, only one study considered the sham group, reporting a lasting reduction in cocaine intake, but no difference between the real and the control groups. ${ }^{87}$ The small sample, however, does not permit any firm conclusion on the efficacy of rTMS in the treatment of cocaine addiction, but results are nevertheless encouraging and foster future investigations. Another study changed the theoretical model by using bursts of pulses (cTBS) over the MPfCx. ${ }^{92}$ This new model started from the hypothesis that the MPfCx is the primary cortical input to the ventral striatum, a central node in elaborating the salience of drug-related stimuli and the motivation/drive for drug seeking. This hypothesis is supported by a previous study, which demonstrated that LTP-like (10 Hz) rTMS to the MPfCx in a group of eleven healthy non-drug-using subjects was associated with a significant reduction in DA-binding potential in the dorsal striatum, reflecting a release of DA in these areas. ${ }^{98}$ Despite the small sample, this new approach could provide a novel efficacious strategy to target the areas involved in craving for cocaine, and reiterates the key role played by impoverished DA transmission in these effects. ${ }^{22}$

The frequency of stimulation observed in the studies revised was $10-20 \mathrm{~Hz}$, with one to 12 sessions. The intensity of stimulation, varied from $90 \%$ to $120 \%$ of MT. In all studies, the targeted area was identified through scalp landmarks, and only one study used neuronavigation to enhance accuracy in targeting the selected brain region to be stimulated. ${ }^{85,99}$ The total number of pulses, a key factor in stimulation efficacy, varied and comprised between 600 and 2,400 pulses/session. ${ }^{100-102}$ For these reasons, all studies reviewed implemented a repetitive-stimulation protocol for several sessions, and this is one of the areas that should be investigated further in future studies to achieve long-lasting effects. ${ }^{39}$ However, none of these studies has been replicated so far and the variability of the stimulation protocol implemented does not allow firm conclusions. Another important factor concerns the measurements of outcome and the followup assessment along the timeline. Half the studies used a self-report scale (VAS) as a measurement for craving. This is a construct developed to define and evaluate the desire/drive to take the substance of abuse reported by the addicts, and thereby it is a subjective measure that correlates with clinical effects and relapse rates. ${ }^{31,33}$ Only two studies of six were objective markers of intake consisting in urine drug tests and hair analysis used. Finally, the timeline of the experimental procedure must be considered. ${ }^{85,87}$ Most of the studies revised evaluated the TMS effects after treatment, and only one study performed 3- and 6-month follow-up. ${ }^{87}$ This aspect must be evaluated carefully for short- and long-term effects so as to modulate the protocol and maximize the stimulation effects.

\section{Conclusion}

We observed encouraging but preliminary evidence of efficacy of rTMS in treating behavioral and psychological symptoms of cocaine addiction. However, the very small samples and lack of methodological control do not permit identification of a specific protocol of stimulation as superior vs others, although the PfCx appears to be the brain area targeted and with solid neurobiological rationale promises to yield more favorable outcomes in terms of reduction of cocaine intake in future..$^{22,44,92}$ Further studies are needed, and should employ standard methodological procedures to find the exact location of targeted areas to improve effectiveness of stimulation, consider subjective (craving) and objective (intake) measurements in relation to neurophysiologic substrates to have a comprehensive understanding of the neuropathology, and consider timeline follow-up to evaluate the lasting nature of neural changes induced by rTMS carefully. Overall, rTMS appears ready to be scrutinized with scientific rigor in a condition (ie, CUD) presently bereft of specific pharmacological and efficacious treatment.

\section{Acknowledgment}

This work was supported in part through a fundraising campaign organized and conducted by Gieffe Supermercati SRL.

\section{Disclosure}

The authors report no conflicts of interest in this work. 


\section{References}

1. European Monitoring Centre for Drugs and Drug Addiction. European Drug Report: Trends and Developments. Lisbon: EMCDDA; 2017.

2. American Psychiatric Association. Diagnostic and Statistical Manual of Mental Disorders. 5th ed. Washington: APA; 2013.

3. Rossetti ZL, Hmaidan Y, Gessa GL. Marked inhibition of mesolimbic dopamine release: a common feature of ethanol, morphine, cocaine and amphetamine abstinence in rats. Eur J Pharmacol. 1992;221:227-234.

4. Melis M, Spiga S, Diana M. The dopamine hypothesis of drug addiction: hypodopaminergic state. Int Rev Neurobiol. 2005;63:101-154.

5. Koob GF, le Moal M. Plasticity of reward neurocircuitry and the 'dark side' of drug addiction. Nat Neurosci. 2005;8:1442-1444.

6. Volkow ND, Wang GJ, Fowler JS, et al. Decreased striatal dopaminergic responsiveness in detoxified cocaine-dependent subjects. Nature. 2007;386:830-833.

7. Volkow ND, Roy AW, Baler R. The dopamine motive system: implications for drug and food addiction. Nat Rev Neurosci. 2017;18:741-752.

8. Jentsch JD, Taylor JR. Impulsivity resulting from frontostriatal dysfunction in drug abuse: implications for the control of behavior by rewardrelated stimuli. Psychopharmacology (Berl). 1999;146:373-390.

9. Goldstein RZ, Volkow ND. Dysfunction of the prefrontal cortex in addiction: neuroimaging findings and clinical implications. Nat Rev Neurosci. 2011;12:652-669.

10. Koob GF, Volkow N. Neurocircuitry of addiction. Neuropsychopharmacology. 2010;35:217-238.

11. Volkow N. Drug dependence and addiction, III: expectation and brain function in drug abuse. Am J Psychiatry. 2004;161:621.

12. Volkow ND, Fowler JS, Wang GJ. The addicted human brain: insights from imaging studies. J Clin Invest. 2003;111:1444-1451.

13. Kalivas PW, Brady K. Getting to the core of addiction: hatching the addiction egg. Nat Med. 2012;18:502-503.

14. Everitt BJ, Robbins TW. Neural systems of reinforcement for drug addiction: from actions to habits to compulsion. Nat Neurosci. 2005;8:1481-1489.

15. Chen J, Tan Z, Zeng L, et al. Heterosynaptic long-term depression mediated by ATP released from astrocytes. Glia. 2013;61:178-191.

16. Goldstein RZ, Volkow ND. Dysfunction of the prefrontal cortex in addiction: neuroimaging findings and clinical implications. Nat Rev Neurosci. 2011;12:652-669.

17. Volkow ND, Fowler JS, Wang GJ, Swanson JM. Dopamine in drug abuse and addiction: results from imaging studies and treatment implications. Mol Psychiatry. 2004;9:557-569.

18. Volkow ND, Fowler JS. Addiction a disease of compulsion and drive: involvement of the orbitofrontal cortex. Cereb Cortex. 2000;10: 318-325.

19. Volkow ND, Koob GF, McLellan AT. Neurobiologic advances from the brain disease model of addiction. N Engl J Med. 2016;374:363-371.

20. London ED. Impulsivity, stimulant abuse and dopamine receptor signaling. Adv Pharmacology. 2016;76:67-84.

21. London ED, Kohno M, Morales AM, Ballard ME. Chronic methamphetamine abuse and corticostriatal deficits revealed by neuroimaging. Brain Res. 2015;1628:174-185.

22. Diana M. The dopamine hypothesis of drug addiction and its potential therapeutic value. Front Psychiatry. 2011;2:64

23. Leggio L, Cardone S, Ferrulli A, et al. Turning the clock ahead: potential preclinical and clinical neuropharmacological targets for alcohol dependence. Curr Pharm Des. 2010;16:2159-218.

24. Volkow ND, Fowler JS, Wang GJ, Baler R, Telang F. Imaging dopamine's role in drug abuse and addiction. Neuropharmacology. 2009;56:3-8.

25. Diana M. The addicted brain. Front Psychiatry. 2013;4:40

26. Kalivas PW. The neural basis of addiction: a pathology of motivation and choice. Am J Psychiatry. 2005;162:1403-1413.

27. Baler RD, Volkow ND. Drug addiction: the neurobiology if disrupted self-control. Trends Mol Med. 2006;12:559-566.
28. Berridge KC. The debate over dopamine's role in reward: the case for incentive salience. Psychopharmacology (Berl). 2007;191:391-431.

29. Everitt BJ, Robbins TW. Neural system of reinforcement for drug addiction: from action to habits to compulsion. Nat Neurosci. 2005;8:1481-1489.

30. Koob GF, le Moal M. Addiction and the brain antireward system. Annu Rev Psychol. 2008;59:29-53.

31. Oslin DW, Cary M, Slaymaker V, Colleran C, Blow FC. Daily ratings measures of alcohol craving during an inpatient stay define subtype of alcohol addiction that predict subsequent risk for resumption of drinking. Drug Alcohol Depend. 2009;103:131-136.

32. Sinha R, Garcia M, Oaliwal O, Kreek MJ, Rounsevill BJ. Stressinduced cocaine craving and hypothalamic-pituitary-adrenal responses are predictive of cocaine relapses outcome. Arch Gen Psychiatry. 2006;63:324-331.

33. Jansen JM, Daams JG, Koeter MW, Veltman DJ, van den Brink W, Goudriaan AE. Effects of non-invasive neurostimulation on craving: a meta-analysis. Neurosci Biobehav Rev. 2013;37:2472-2480.

34. Fattore L, Diana M. Drug addiction: an affective-cognitive disorder in need of a cure. Neurosci Biobehav Rev. 2016;65:341-361.

35. Garavan HH. The role of cognitive control in cocaine dependence. Neuropsychol Rev. 2007;17:337-345.

36. van Holst RJ, Schilt T. Drug-related decrease in neuropsychological functions of abstinent drug users. Curr Drug Abuse Rev. 2011;4: $42-56$.

37. Back SE, Hartwell K, DeSantis SM, et al. Reactivity to laboratory stress provocation predicts relapse to cocaine. Drug Alcohol Depend. 2010;106:21-27.

38. Poling J, Kosten TR, Sofuoglu M. Treatment outcome predictors for cocaine dependence. Am J Drug Alcohol Abuse. 2007;33:191-206.

39. Feil J, Zangen A. Brain stimulation in the study and treatment of addiction. Neurosci Biobehav Rev. 2010;34:559-574.

40. Sundaresan K, Ziemann U, Stan-Ley J, Boutros N. Cortical inhibition and excitation in abstinent cocaine-dependent patients: a transcranial magnetic stimulation study. Neuroreport. 2007;18:289-292.

41. Barker A, Jalinous R, Freeston I. Non-invasive magnetic stimulation of human motor cortex. Lancet. 1985;1:1106-1107.

42. Di Lazzaro V, Ziemann U, Lemon R. State of the art: physiology of transcranial motor cortex stimulation. Brain Stimul. 2008;1:345-362.

43. Gorelick DA, Zangen A, George MS. Transcranial magnetic stimulation in the treatment of substance addiction. Ann N Y Acad Sci. 2014;1327:79-93.

44. Diana M, Raij T, Melis M, Nummenmaa A, Leggio L, Bonci A. Rehabilitating the addicted brain with transcranial magnetic stimulation. Nat Rev Neurosci. 2017;18:685-693.

45. Lisanby SH, Husain MM, Rosenquist PB, et al. Daily left prefrontal repetitive transcranial magnetic stimulation in the acute treatment of major depression: clinical predictors of outcome in a multisite, randomized controlled clinical trial. Neuropsychopharmacology. 2009;34:522-534.

46. Benadhira R, Thomas F, Bouaziz N, et al. A randomized, shamcontrolled study of maintenance rTMS for treatment-resistant depression (TRD). Psychiatry Res. 2017;258:226-233.

47. Fitzgerald PB, Hoy KE, Herring SE, et al. A double blind randomized trial of unilateral left and bilateral prefrontal cortex transcranial magnetic stimulation in treatment resistant major depression. J Affect Disord. 2012;139:193-198.

48. Alonso P, Pujol J, Cardoner N, et al. Right prefrontal repetitive transcranial magnetic stimulation in obsessive-compulsive disorder: a double-blind, placebo-controlled study. Am J Psychiatry. 2001;158: $1143-1145$.

49. Lee SH, Kim W, Chung YC, et al. A double blind study showing that two weeks of daily repetitive TMS over the left or right temporoparietal cortex reduces symptoms in patients with schizophrenia who are having treatment-refractory auditory hallucinations. Neurosci Lett. 2005;376:177-181 
50. Jin Y, Potkin SG, Kemp AS, et al. Therapeutic effects of individualized alpha frequency transcranial magnetic stimulation ( $\alpha$ TMS) on the negative symptoms of schizophrenia. Schizophr Bull. 2005;32:556-561.

51. Rossi S, Hallett M, Rossini PM, Pascual-Leone A. Safety, ethical considerations, and application guidelines for the use of transcranial magnetic stimulation in clinical practice and research. Clin Neurophysiol. 2009;120:2008-2039.

52. Paus T, Jech R, Thompson CJ, et al. Transcranial magnetic stimulation during positron emission tomography: a new method for studying connectivity of the human cerebral cortex. J Neurosci. 1997; 17:3178-3184.

53. Ilmoniemi RJ, Virtanen J, Ruohonen J, et al. Neuronal responses to magnetic stimulation reveal cortical reactivity and connectivity. Neuroreport. 1997;8:3537-3540.

54. Terao Y, Ugawa Y. Basic mechanisms of TMS. J Clin Neurophysiol. 2002;19:322-343.

55. Roth Y, Amir A, Levkovitz Y, Zangen A. Three-dimensional distribution of the electric field induced in the brain by TMS using figure- 8 and deep H-coils. J Clin Neurophysiol. 2007;24:31-38.

56. Hoogendam J, Ramakers G, Di Lazzaro V. Physiology of repetitive transcranial magnetic stimulation of the human brain. Brain Stimul. 2010;3:95-118.

57. Ceccanti M, Inghilleri M, Attilia ML, et al. Deep TMS on alcoholics: effects on cortisolemia and dopamine pathway modulation - a pilot study. Can J Physiol Pharmacol. 2015;93:283-290.

58. Mishra BR, Nizamie SH, Das B, Praharaj SK. Efficacy of repetitive transcranial magnetic stimulation in alcohol dependence: a shamcontrolled study. Addiction. 2010;105:49-55.

59. Azevedo CA, Mammis AM. Neuromodulation therapies for alcohol addiction: a literature review. Neuromodulation. 2018;21:144-148.

60. Amiaz R, Levy D, Vainiger D, Grunhaus L, Zangen A. Repeated high-frequency transcranial magnetic stimulation over the dorsolateral prefrontal cortex reduces cigarette craving and consumption. Addiction. 2009;104:653-660.

61. Dinur-Klein L, Dannon P, Hadar A, et al. Smoking cessation induced by deep repetitive transcranial magnetic stimulation of the prefrontal and insular cortices: a prospective, randomized controlled trial. Biol Psychiatry. 2014;76:742-749.

62. Li X, Malcolm RJ, Huebner K, et al. Low frequency repetitive transcranial magnetic stimulation of the left dorsolateral prefrontal cortex transiently increases cue-induced craving for methamphetamine: a preliminary study. Drug Alcohol Depend. 2013;133:641-646.

63. Shen $\mathrm{Y}, \mathrm{Cao} \mathrm{X}$, Tan $\mathrm{T}$, et al. $10-\mathrm{Hz}$ repetitive transcranial magnetic stimulation of the left dorsolateral prefrontal cortex reduces heroin cue craving in long-term addicts. Biol Psychiatry. 2016;80:e13-e14.

64. Makani R, Pradhan B, Shah U, Parikh T. Role of repetitive transcranial magnetic stimulation (rTMS) in treatment of addiction and related disorders: a systematic review. Curr Drug Abuse Rev. Epub 2017 Nov 29.

65. Spagnolo PA, Goldman D. Neuromodulation interventions for addictive disorders: challenges, promise, and roadmap for future research. Brain. 2017;140:1183-1203.

66. Strafella AP, Paus T, Barrett J, Dagher A. Repetitive transcranial magnetic stimulation of the human prefrontal cortex induces dopamine release in the caudate nucleus. J Neurosci. 2001;21:RC157.

67. Cho S, Strafella A. rTMS of the left dorsolateral prefrontal cortex modulates dopamine release in the ipsilateral anterior cingulate cortex and orbitofrontal cortex. PLoS One. 2009;4:e6725.

68. Goldstein RZ, Leskovjan AC, Hoff AL, et al. Severity of neuropsychological impairment in cocaine and alcohol addiction: association with metabolism in the prefrontal cortex. Neuropsychologia. 2004;42:1447-1458.

69. Roth Y, Zangen A, Hallett M. A coil design for transcranial magnetic stimulation of deep brain regions. J Clin Neurophysiol. 2002;19:361-370.

70. Roth Y, Zangen A, Voller B, Hallett M. Transcranial magnetic stimulation of deep brain regions: evidence for efficacy of the H-coil. Clin Neurophysiol. 2005;116:775-779.
71. Roth Y, Pell GS, Chistyakov AV, Sinai A, Zangen A, Zaaroor M. Motor cortex activation by H-coil and figure- 8 coil at different depths: combined motor threshold and electric field distribution study. Clin Neurophysiol. 2014;125:336-343.

72. Nummenmaa A, Stenroos M, Ilmoniemi RJ, Okada YC, Hämäläinen MS, Raij T. Comparison of spherical and anatomically realistic boundary element models for transcranial magnetic stimulation navigation. Clin Neurophysiol. 2013;124:1995-2007.

73. Deng Z, Lisanby S, Peterchev A. Electric field depth-focality tradeoff in transcranial magnetic stimulation: simulation comparison of 50 coil designs. Brain Stimul. 2013;6:1-13.

74. Rossini PM, Burke D, Chen R, et al. Non-invasive electrical and magnetic stimulation of the brain, spinal cord, roots and peripheral nerves: basic principles and procedures for routine clinical and research application - an updated report from an I.F.C.N. Committee. Clin Neurophysiol. 2015;126:1071-1107.

75. Zangen A, Roth Y, Voller B, Hallett M. Transcranial magnetic stimulation of deep brain regions: evidence for efficacy of the H-coil. Clin Neurophysiol. 2005;116:775-779.

76. Malik S, Jacobs M, Cho SS, et al. Deep TMS of the insula using the $\mathrm{H}$-coil modulates dopamine release: a crossover $\left[{ }^{11} \mathrm{C}\right] \mathrm{PHNO}-\mathrm{PET}$ pilot trial in healthy humans. Brain Imaging Behav. Epub 2017 Nov 23.

77. Salling MC, Martinez D. Brain stimulation in addiction. Neuropsychopharmacology. 2016;41:2798-2809.

78. Ruohonen J, Karhu J. Navigated transcranial magnetic stimulation. Neurophysiol Clin. 2010;40:7-17.

79. Camprodon JA, Martinez-Raga J, Alonso-Alonso M, Shih MC, Pascual-Leone A. One session of high frequency repetitive transcranial magnetic stimulation (rTMS) to the right prefrontal cortex transiently reduces cocaine craving. Drug Alcohol Depend. 2007;86:91-94.

80. Robertson EM, Theoret H, Pascual-Leone A. Studies in cognition: the problems solved and created by transcranial magnetic stimulation. $J$ Cogn Neurosci. 2003;15:948-960.

81. Politi E, Fauci E, Santoro A, Smeraldi E. Daily sessions of transcranial magnetic stimulation to the left prefrontal cortex gradually reduce cocaine craving. Am J Addict. 2008;17:345-346.

82. Maas LC, Lukas SE, Kaufman MJ, et al. Functional magnetic resonance imaging of human brain activation during cue-induced cocaine craving. Am J Psychiatry. 1998;155:124-126.

83. Garavan H, Pankiewicz J, Bloom A, et al. Cue-induced cocaine craving: neuroanatomical specificity for drug users and drug stimuli. Am J Psychiatry. 2000;157:1789-1798.

84. Bonson KR, Grant SJ, Contoreggi CS, et al. Neural systems and cue induced cocaine craving. Neuropsychopharmacology. 2002;26: 376-386.

85. Terraneo A, Leggio L, Saladini M, Ermani M, Bonci A, Gallimberti L. Transcranial magnetic stimulation of dorsolateral prefrontal cortex reduces cocaine use: a pilot study. Eur Neuropsychopharmacol. 2016;26:37-44.

86. Rapinesi C, del Casale A, Di Pietro S, et al. Add-on high frequency deep transcranial magnetic stimulation (dTMS) to bilateral prefrontal cortex reduces cocaine craving in patients with cocaine use disorder. Neurosci Lett. 2016;629:43-47.

87. Bolloni C, Panella R, Pedetti M, et al. Bilateral transcranial magnetic stimulation of the prefrontal cortex reduces cocaine intake: a pilot study. Front Psychiatry. 2016;7:133.

88. Levkovitz Y, Harel EV, Roth Y, et al. Deep transcranial magnetic stimulation over the prefrontal cortex: evaluation of antidepressant and cognitive effects in depressive patients. Brain Stimul. 2009;2:188-200.

89. Levkovitz Y, Isserless M, Padberg F, et al. Efficacy and safety of deep transcranial magnetic stimulation for major depression: a prospective multicenter randomized controlled trial. World Psychiatry. 2015;14:64-73.

90. Ursitti F, Klein J, Sellers E, Koren G. Use of hair analysis for confirmation of self-reported cocaine use in users with negative urine. JToxicol Clin Toxicol. 2001;39:361-366. 
91. DuPont RL, Baumgartner WA. Drug testing by urine and hair analysis: complementary features and scientific issue. Forensic Sci Int. 1995;70:63-76.

92. Hanlon CA, Dowdle LT, Austelle CW, et al. What goes up, can come down: novel brain stimulation paradigms may attenuate craving and craving related neural circuitry in substance dependent individuals. Brain Res. 2015;1628:199-209.

93. Bear MF, Malenka RC. Synaptic plasticity: LTP and LTD. Curr Opin Neurobiol. 1994;4:389-399.

94. Malenka RC, Bear MF. LTP and LTD: an embarrassment of riches. Neuron. 2004;44:5-21.

95. Huang YZ, Edwards MJ, Rounis E, Bhatia KP, Rothwell JC. Theta-burst stimulation of the human motor cortex. Neuron. 2005;45:201-206.

96. Di Lazzaro V, Pilato F, Saturno E, et al. Theta-burst repetitive transcranial magnetic stimulation suppresses specific excitatory circuits in the human motor cortex. J Physiol. 2005;565:945-950.

97. Hanlon CA, Dowdle LT, Moss H, Canterberry M, George MS. Mobilization of medial and lateral frontal-striatal circuits in cocaine users and controls: an interleaved TMS/BOLD functional connectivity study. Neuropsychopharmacol. 2016;41:3032-3041.

98. Sinha R. New findings on biological factors predicting addiction relapse vulnerability. Curr Psychiatry Rep. 2011;13:398-405.

99. Wing VC, Barr MS, Wass CE, et al. Brain stimulation methods to treat tobacco addiction. Brain Stimul. 2013;6:221-230.

100. Cho SS, Koshimori Y, Aminian K, et al. Investing in the future: stimulation of the medial prefrontal cortex reduces discounting of delayed rewards. Neuropsychopharmacology. 2015;40:546-553.

101. Fitzgerald PB, Hoy K, McQueen S, Maller JJ, Herring S, Segrave R. A randomized trial of rTMS targeted with MRI based neuro-navigation in treatment-resistant depression. Neuropsychopharmacology. 2009;34:1255-1262.

102. Gershon AA, Dannon PN, Grunhaus L. Transcranial magnetic stimulation in the treatment of depression. Am J Psychiatry. 2003;160:835-845.

103. Johann M, Wiegand R, Kharraz A, et al. [Transcranial magnetic stimulation for nicotine dependence]. Psychiatr Prax. 2003;30:S129 S131. German.

104. Li X, Hartwell KJ, Owens M, et al. Repetitive transcranial magnetic stimulation of the dorsolateral prefrontal cortex reduces nicotine cue craving. Biol Psychiatry. 2013;73:714-720.

105. Eichhammer P, Johann M, Kharraz A, et al. High-frequency repetitive transcranial magnetic stimulation decreases cigarette smoking. J Clin Psychiatry. 2003;64:951-953.

106. Pripfl J, Tomova L, Riecansky I, Lamm C. Transcranial magnetic stimulation of the left dorsolateral prefrontal cortex decreases cue-induced nicotine craving and EEG delta power. Brain Stimul. 2014;7:226-233.
107. Hayashi T, Ko JH, Strafella AP, Dagher A. Dorsolateral prefrontal and orbitofrontal cortex interactions during self-control of cigarette craving. Proc Natl Acad Sci U S A. 2013;110:4422-4427.

108. Rose JE, McClernon FJ, Froeliger B, Behm FM, Preud'homme X, Krystal AD. Repetitive transcranial magnetic stimulation of the superior frontal gyrus modulates craving for cigarettes. Biol Psychiatry. 2011;70:794-799.

109. Wing VC, Bacher I, Wu BS, Daskalakis ZJ, George TP. High frequency repetitive transcranial magnetic stimulation reduces tobacco craving in schizophrenia. Schizophr Res. 2012;139:264-266.

110. Prikryl R, Ustohal L, Kucerova HP, et al. Repetitive transcranial magnetic stimulation reduces cigarette consumption in schizophrenia patients. Prog Neuropsychopharmacol Biol Psychiatry. 2014;49:30-35.

111. Trojak B, Meille V, Achab S, et al. Transcranial magnetic stimulation combined with nicotine replacement therapy for smoking cessation: a randomized controlled trial. Brain Stimul. 2015;8:1168-1174.

112. Addolorato G, Antonelli M, Cocciolillo F. Deep transcranial magnetic stimulation of the dorsolateral prefrontal cortex in alcohol use disorder patients: effects on dopamine transporter availability and alcohol intake. Eur Neuropsychopharmacol. 2017;279:450-461.

113. Mishra BR, Praharaj SK, Katshu MZ, Sarkar S, Nizamie SH. Comparison of anticraving efficacy of right and left repetitive transcranial magnetic stimulation in alcohol dependence: a randomized doubleblind study. J Neuropsychiatry Clin Neurosci. 2015;27:e54-e59.

114. Herremans SC, Baeken C, Vanderbruggen N, et al. No influence of one right sided prefrontal HF-rTMS session on alcohol craving in recently detoxified alcohol-dependent patients: results of a naturalistic study. Drug Alcohol Depend. 2012;120:209-213.

115. Herremans SC, Vanderhasselt MA, de Raedt R, Baeken C. Reduced intra-individual reaction time variability during a Go-NoGo task in detoxified alcohol-dependent patients after one right-sided dorsolateral prefrontal HF-rTMS session. Alcohol Alcohol. 2013;48:552-557.

116. Herremans SC, van Schuerbeek P, de Raedt R, et al. The impact of accelerated right prefrontal high-frequency repetitive transcranial magnetic stimulation (rTMS) on cue-reactivity: an fMRI study on craving in recently detoxified alcohol-dependent patients. PLoS One. 2015;10:e0136182.

117. Höppner J, Broese T, Wendler L, Berger C, Thome J. Repetitive transcranial magnetic stimulation (rTMS) for treatment of alcohol dependence. World J Biol Psychiatry. 2011;12:57-62.

118. del Felice A, Bellamoli E, Formaggio E, et al. Neurophysiological, psychological and behavioural correlates of rTMS treatment in alcohol dependence. Drug Alcohol Depend. 2016;158:147-153.

119. Su H, Zhong N, Gan H, et al. High frequency repetitive transcranial magnetic stimulation of the left dorsolateral prefrontal cortex for methamphetamine use disorders: a randomised clinical trial. Drug Alcohol Depend. 2017;175:84-91. 


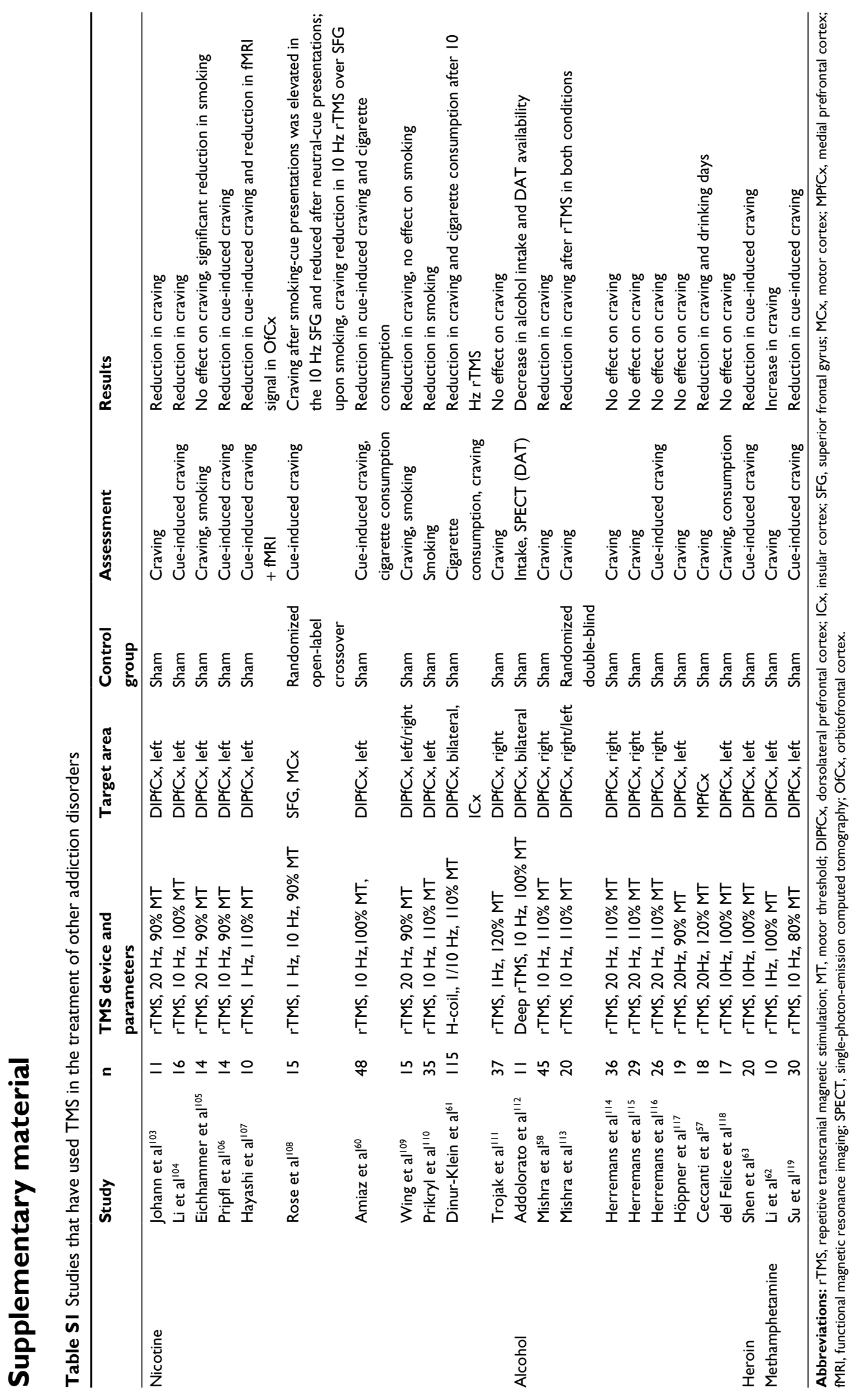


Substance Abuse and Rehabilitation is an international, peer-reviewed, open access journal publishing original research, case reports, editorials, reviews and commentaries on all areas of addiction and substance abuse and options for treatment and rehabilitation. The manuscript management system is completely online and includes a very quick and fair peer-review system. Visit http://www.dovepress.com/testimonials.php to read real quotes from published authors.

Submit your manuscript here: https://www.dovepress.com/substance-abuse-and-rehabilitation-journal 